Review Article

\title{
Pretreatment Lymphocyte Monocyte Ratio Predicts Long-Term Outcomes in Patients with Digestive System Tumor: A Meta-Analysis
}

\author{
Jingwen Zhang, ${ }^{1}$ Lishan Chen, ${ }^{2}$ Rui Zhou, ${ }^{1}$ Huiying Sun, ${ }^{1}$ Yulin Liao, ${ }^{3}$ and Wangjun Liao ${ }^{1}$ \\ ${ }^{1}$ Department of Oncology, Nanfang Hospital, Southern Medical University, Guangzhou 510515, China \\ ${ }^{2}$ Huiqiao Medical Center, Nanfang Hospital, Southern Medical University, Guangzhou 510515, China \\ ${ }^{3}$ Department of Cardiology, Nanfang Hospital, Southern Medical University, Guangzhou 510515, China \\ Correspondence should be addressed to Wangjun Liao; nfyyliaowj@163.com
}

Received 29 February 2016; Revised 13 June 2016; Accepted 5 July 2016

Academic Editor: Roberto César P. Lima-Júnior

Copyright (C) 2016 Jingwen Zhang et al. This is an open access article distributed under the Creative Commons Attribution License, which permits unrestricted use, distribution, and reproduction in any medium, provided the original work is properly cited.

\begin{abstract}
Purpose. The prognostic value of pretreatment lymphocyte monocyte ratio (LMR) in digestive system cancer patients remains controversial. The aim of this study was to quantify the prognostic impact of this biomarker and assess its consistency in digestive system tumors. Methods. We searched "PubMed," "Embase," and "CBM" for published eligible studies before June 2016 and conducted a meta-analysis to estimate the pooled hazard ratios (HRs) for disease recurrence and mortality focusing on LMR. Subgroup analyses, meta-regression, and sensitivity analyses were also performed. Results. A total of 22 cohort studies enrolling 12829 patients with digestive system cancer were included. The summary results showed that lower LMR was significantly associated with worse overall survival (OS), cancer-specific survival (CSS), and tumor disease or recurrence-free survival (DFS/RFS) in analyses using the studies reporting HRs either by the univariate analyses ( $\mathrm{HR}=1.32, \mathrm{HR}=1.35$, and $\mathrm{HR}=1.26 \mathrm{for}$ OS, CSS, and DFS/RFS, resp.) or by multivariate analyses ( $\mathrm{HR}=1.21, \mathrm{HR}=1.18$, and $\mathrm{HR}=1.26$ for OS, CSS, and DFS/RFS, resp.). Conclusion. Our results support the fact that decreased LMR indicates worse prognosis in multiple digestive system tumors.
\end{abstract}

\section{Introduction}

There is increasing evidence showing that the tumor microenvironment (TME) and TME-related pathways weigh a lot in tumor growth, invasion, and metastasis $[1,2]$. Indeed, the crosstalk between tumor cells and their ambient TME determines the outcome of these biological processes [3]. Recently, the close relationship between systemic inflammatory response, an important component of TME, and cancer development has been gradually taken into concern [4]. Inflammation regulating factors and effector cells are shown to take part in various carcinogenetic events [5]. Meanwhile, some peripheral blood inflammatory parameters are found to have prognostic prediction values in cancer patients $[6,7]$. Obviously, compared with immunohistochemical markers that largely depend on the resection or biopsy of tumor samples, obtaining peripheral blood samples is convenient, less invasive, and easier for dynamic evaluation. Among these markers, the pretreatment lymphocyte monocyte ratio (LMR) in particular has gained notable interest recently. There has been only two meta-analyses which revealed that an elevation of LMR was likely to indicate a better prognosis in various solid tumors $[8,9]$. However, the consistency and magnitude of the prognostic impact of LMR, especially in digestive system tumors, still lack systematic analyses to confirm. Accordingly, we conducted a systematic review and meta-analysis in the hope of identifying the clinical value of pretreatment LMR elevation in predicting long-term outcomes for digestive system tumors.

\section{Materials and Methods}

2.1. Literature Search and Study Selection. Systematic computerized search of PubMed, Embase, and Chinese Biomedical Literature Database (CBM) was conducted in June 2016. The following keywords were used in various forms and 
combinations for "Title/Abstract" based search: "cancer", "tumor", "carcinoma", "neoplasm", "adenocarcinoma", "malignant”, "oncology”, "lymphocyte monocyte ratio", and "monocyte lymphocyte ratio". Additionally, "neoplasm" was also used in "Medical Subject Headings" based search. An example of initial search strategy using recognized search terms was provided in Supplementary Material (see Supplementary Material available online at http://dx.doi.org/10.1155/ 2016/9801063). References listed within selected studies were also searched for potentially eligible studies. Corresponding authors were contacted for further information if necessary. Study was conducted according to the Preferred Reporting Items for Systematic Reviews and Meta-Analyses (PRISMA) statement [10].

The abstracts of all candidate articles were read by two independent reviewers (Zhang and Chen). Articles that could not be categorized based on title and abstract alone were retrieved for full-text review. These articles were independently read and checked for inclusion criteria. Any disagreements were resolved through consensus with a third reviewer (Zhou).

2.2. Study Inclusion/Exclusion Criteria. Inclusion criteria for primary studies were as follows: (a) patients were diagnosed as digestive system tumors; (b) more than 10 patients were involved; (c) the correlation between pretreatment LMR value and survival information was investigated; (d) the study was original; (e) the study was published as a full-text paper in either English or Chinese.

Studies were excluded based on the following criteria: (a) letters, reviews, case reports, editorials, expert opinion, or laboratory studies; (b) studies that had duplicate data or repeat analysis; (c) cancer treatments prior to obtaining peripheral blood samples; (d) lacking of key information for further analysis, such as survival information; (e) nonhuman research.

2.3. Quality Assessment of Primary Studies. The quality assessment of the included studies was performed by the three primary reviewers (Zhang, Chen, and Zhou) independently. The quality of all the 22 acceptable studies elaborating LMR and survival information was evaluated using the NewcastleOttawa Quality Assessment Scale (NOS) for cohort studies (Table S1). The modified NOS (http://www.ohri.ca/programs/ (clinical_epidemiology/oxford.asp) of non-RCTs addressed the following three items: patient selection, comparability of groups, and outcome assessment. Stars were given to highquality elements and the total was used for study quality comparison in a quantitative manner. We considered a study awarded seven or more stars as a high-quality study [11].

2.4. Data Extraction. Data were independently extracted from the eligible articles by two reviewers (Zhang and Chen). The following data were collected from each of the studies: study characteristics (author name, year of publication, and number of patients), patient characteristics (age, gender, and country), tumor site, anatomic structure type, distant metastatic status, treatment strategy, the lower limit of elevated LMR, the portion of patients whose LMR values were higher than cut-off, and survival information (follow-up months, whether multivariate analysis (MVA) was conducted, and the method of getting univariate analysis (UVA) hazard ratio (HR)).

If HRs and their confidence intervals (CIs) were not directly reported but the information of the number of patients with high and low LMR levels together with the number of observed deaths or disease recurrences was available, mathematical HR was estimated using the established method [12]. In the case when sufficient data were not directly available but a Kaplan-Meier curve was provided, we estimated HR through the extracted data from the KaplanMeier curve using the same method [12]. If none of the above information was reported, the study then was excluded.

2.5. Descriptive Statistics and Meta-Analysis. We performed statistical analysis using RevMan software version 5.1 (Cochrane Collaboration, Oxford, UK) and the META module of STATA version 12.0 (Stata Corporation, College Station, TX). Considering the association between LMR and clinicopathological variables and that such relativity might also be one of the reasons why LMR level influences patient prognosis, we conducted our meta-analysis using HRs from UVA and MVA separately.

For survival analysis, the pooled HRs were calculated. An observed HR $>1$ indicated worse outcome for the study group relative to the reference group. Heterogeneity among studies was examined using $I^{2}$ [13]. Substantial heterogeneity was defined as an $I^{2}>50 \%$. A fixed-effects model was applied when $I^{2}<50 \%$; otherwise a random-effects model was used. The prespecified subgroup analyses were performed using the following variables: anatomic structure, cancer type, disease stage, patient ethnicity, cut-off value used, and treatment strategy. Meta-regression analyses were used to explore the potential heterogeneity contributors $[14,15]$. In order to avoid data dredging, we firstly conducted meta-regression focusing on one single covariate at a time to figure out significant covariates. If there was more than one significant covariate, we then adjust them in corresponding models simultaneously to further identify independent covariates. Sensitivity analyses were also conducted by changing the effect models or estimating the average HR after sequential omission of each individual study. Publication bias was evaluated by Egger's test [16]. When the publication bias was observed, the trim and fill method was used to test the stability of the results [17]. Statistical significance was reached when $P$ values $<0.05$.

\section{Results}

3.1. Literature Search and Quality Assessment. The titles and abstracts of 322 primary studies were identified for initial review using searching strategies as described and no additional records were identified through the references listed within selected studies. A total of 22 studies [18-39] were finally included for systematic review following the PRISMA statement (Figure 1). The selection and quality assessment was performed on all 22 studies. The total stars of each of the studies were all more than seven stars, suggesting acceptable 


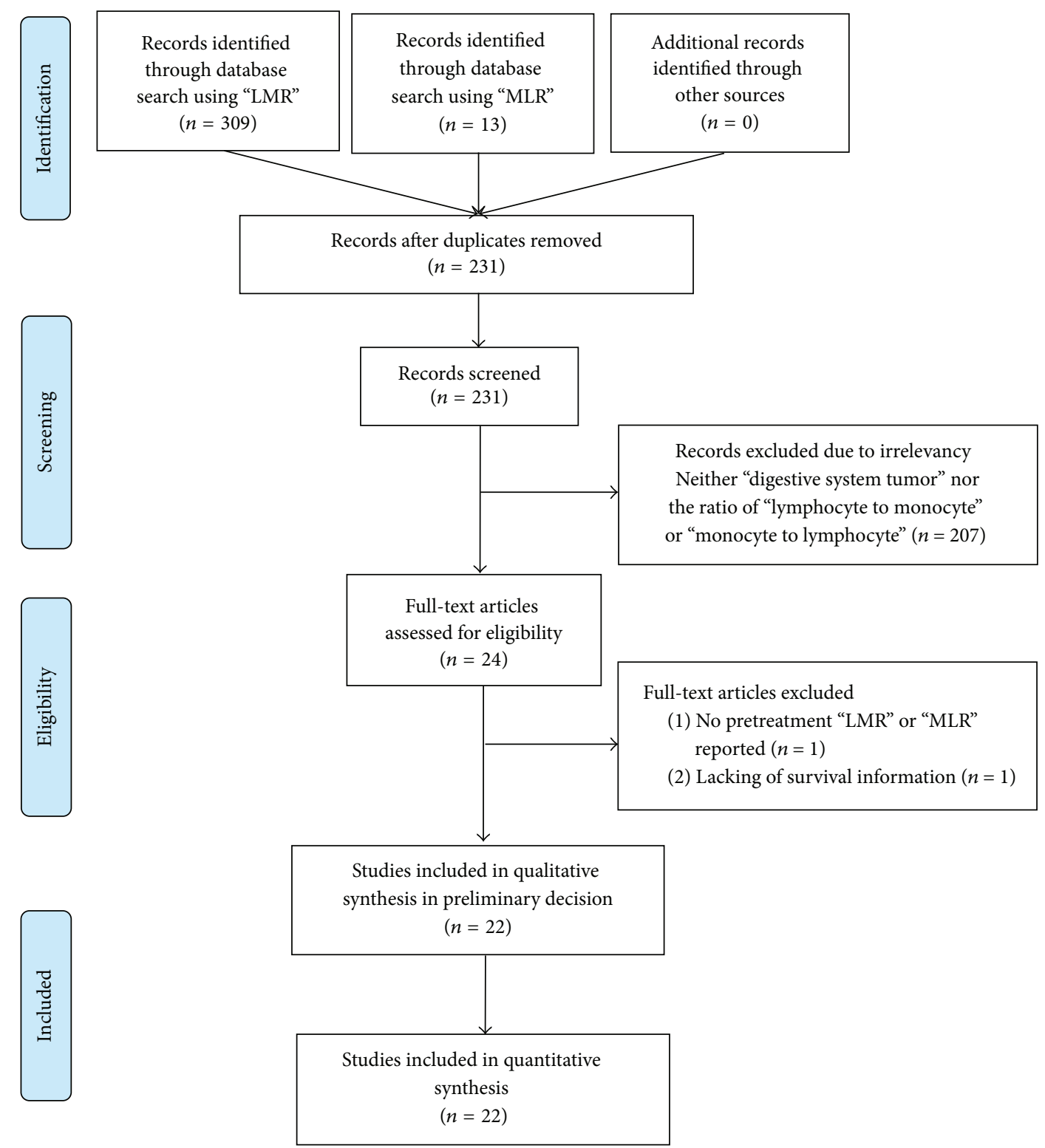

FIGURE 1: Selection of studies included in the analysis. LMR: lymphocyte to monocyte ratio.

overall quality of the included studies. The results of selection and quality assessment were listed in Table S2.

3.2. Main Study Characteristics. A total of 12829 patients were included for the meta-analysis. Characteristics of included studies were shown in Table 1 and Table S3. In these studies, the lower limit of elevated LMR ranged from 2.3 to 4.95 . The UVA HRs and their 95\% CIs of included studies were collected, and ten of them from six studies were estimated using Kaplan-Meier survival curves, while others were directly reported. All studies we included conducted multivariable analysis except the study of Neal et al. [24].

3.3. Meta-Analysis of HRs from Univariate Analysis. All studies were included in the meta-analyses for survival based on UVA HRs. Eighteen studies reported overall survival
(OS), six studies reported cancer-specific survival (CSS), and thirteen studies reported disease or recurrence-free survival (DFS/RFS). The main results were listed in Table 2.

Through meta-analyses, we found that decreased LMR indicated not only a higher risk for patients overall mortality (HR 1.32, 95\% CI 1.28-1.36; $I^{2} 0$, Figure 2(a)), but also cancerspecific death (HR 1.35, 95\% CI 1.20-1.50; $I^{2}$ 65\%, Figure 2(b)) and tumor recurrence (HR 1.26, 95\% CI 1.18-1.33; $I^{2} 63 \%$, Figure 2(c)). Then subgroup analyses (Table 2) revealed that the significantly improved prognostic effect of lower pretreatment LMR for OS could be observed in all tumor sites (the largest effect size was observed in the patients with liver cancer), in cancers of anatomic structure of gastrointestinal tract or nongastrointestinal tract, in Asian or nonAsian patients, in non-metastasis, metastasis, or mixed tumor stages, and in patients receiving surgery or not. A separate 


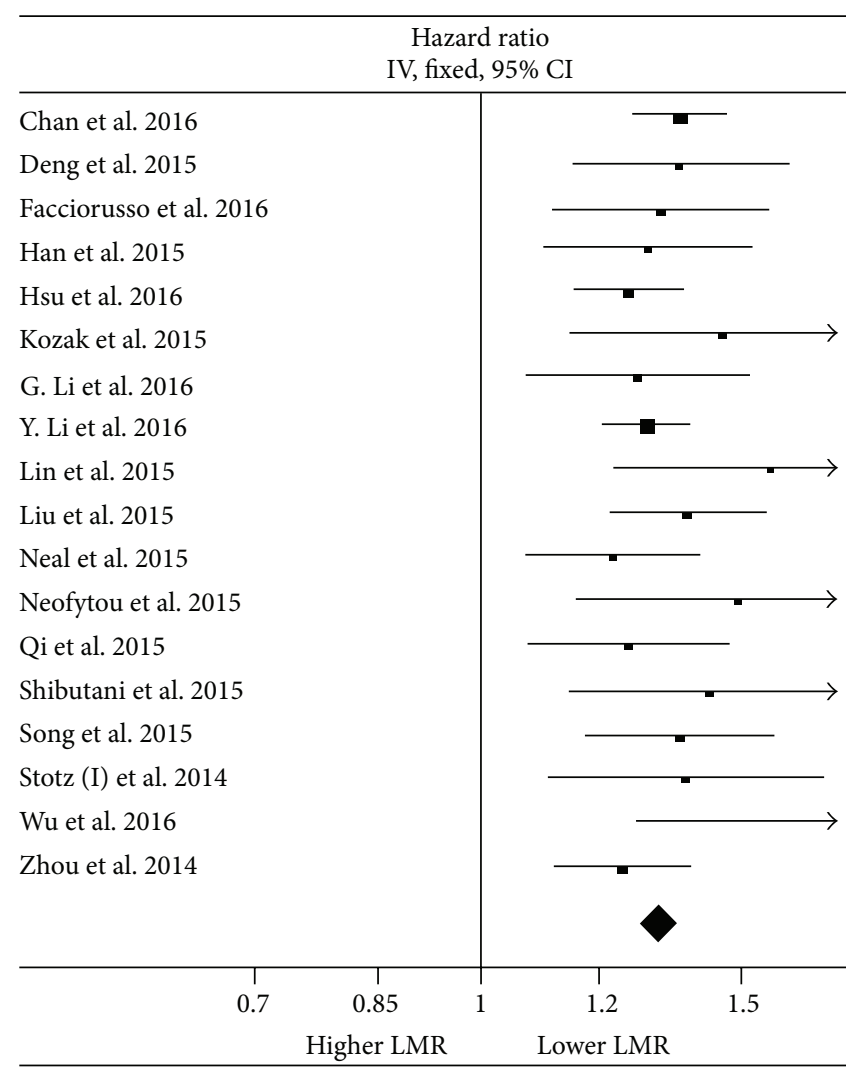

(a)

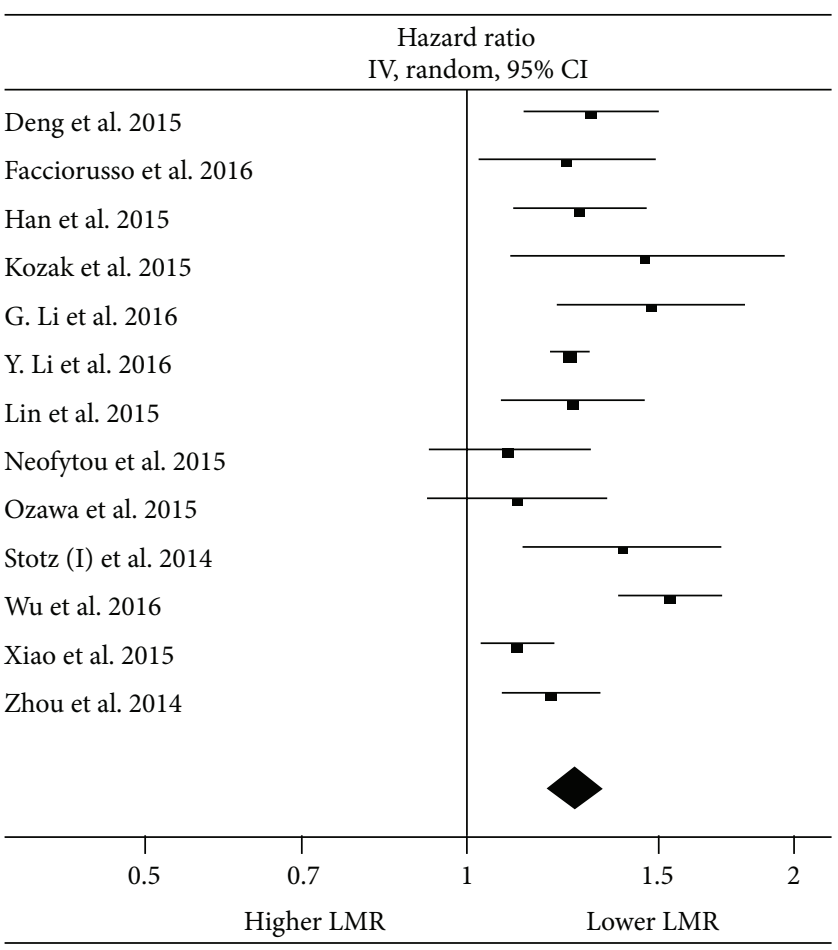

(c)

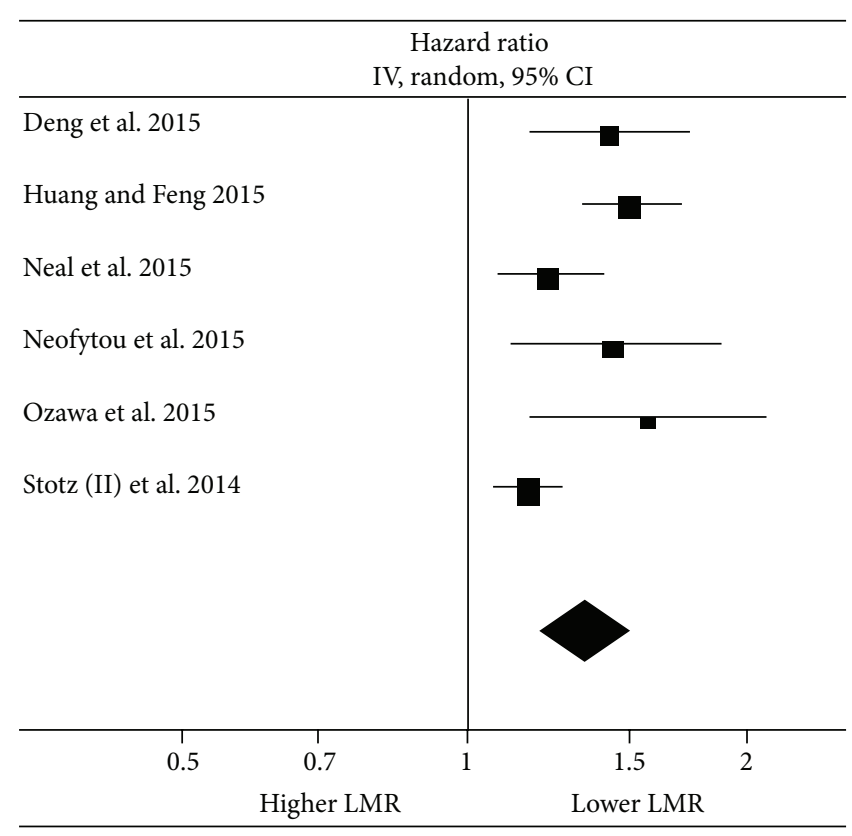

(b)

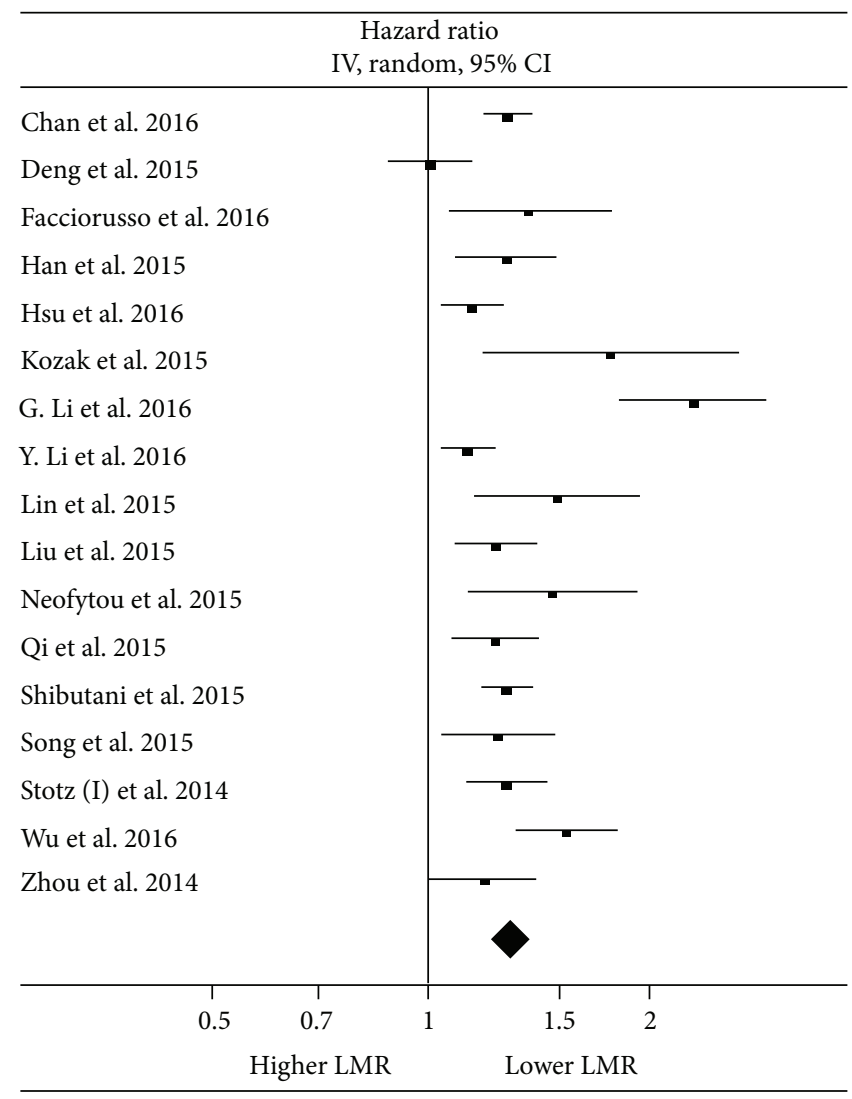

(d)

Figure 2: Continued. 


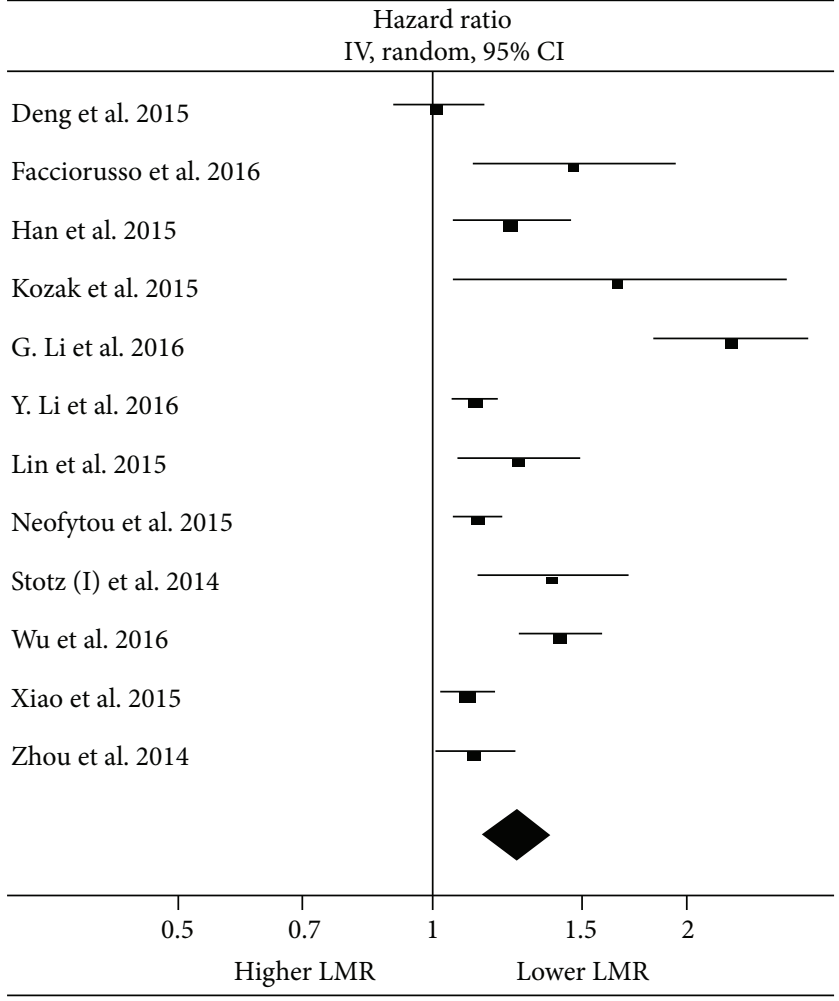

(f)

FIGURE 2: Main forest plots of meta-analysis. Forest plots of the association between lymphocyte to monocyte ratio and overall survival (OS), cancer-specific survival (CSS), and disease or recurrence-free survival (DFS/RFS). Hazard ratios (HRs) for each study are represented by the squares. The size of the data markers (squares) corresponds to the weight of the study in the meta-analysis, and the horizontal line crossing the square represents the $95 \%$ confidence interval (CI). All statistical tests were two-sided. (a) Meta-analysis of patients OS from univariate analysis (UVA); (b) meta-analysis of patients CSS from UVA; (c) meta-analysis of patients DFS/RFS from UVA; (d) meta-analysis of patients OS from multivariate analysis (MVA); (e) meta-analysis of patients CSS from MVA; (f) meta-analysis of patients DFS/RFS from MVA.

analysis performed on cut-off values also identified lower LMR value as an unfavorable factor for improved prognosis in subgroups of the data applying " $<3.0$ " or " $\geq 3.0$ ". Concerning subgroup analyses of CSS and DFS (Table 2), the significant survival benefit was also observed in all subgroups regarding tumor site, anatomic structure, tumor stage, ethnicity, and cut-off value.

3.4. Meta-Analysis of HRs from Multivariate Analysis. MVA were performed in 21 studies. In meta-analysis of MVA HRs (Table 3), LMR less than the cut-off was associated with HRs for OS of 1.29 (95\% CI 1.21-1.38; I $I^{2} 73 \%$, Figure 2(d)), for CSS of 1.18 (95\% CI 1.04-1.34; $I^{2} 65 \%$, Figure 2(e)), and for DFS/RFS of 1.26 (95\% CI 1.16-1.38; $I^{2} 83 \%$, Figure 2(f)). In subgroup analyses for OS, the decrease of OS in patients with lower LMR reached significance in all subgroups except in patients with pancreatic cancer. In terms of subgroup analyses for CSS, however, the LMR did not appear to be an independent prognostic factor in patients with mixed stage, in both subgroups according to patient ethnicity and in the LMR cut-off value $\geq 3.0$ group. As for analyses of $\mathrm{DFS} / \mathrm{RFS}$, the independent positive prognostic effect of a low
LMR on DFS/RFS was not seen in subgroups of gastric cancer patients. Meta-regression analyses showed that the tumor site and the anatomic structure type were observed to be heterogeneity contributors for both OS and DFS/RFS through meta-regressions based on single variables. When combining both tumor site and the anatomic structure type simultaneously, only the anatomic structure type maintained its significance for OS $(P=0.016)$, while the anatomic structure type $(P=0.015)$ and tumor site of liver $(P=0.018)$ still contributed to heterogeneity of DFS/RFS significantly independently. None of other variables were found to be potential sources of heterogeneity (Table 4).

3.5. Sensitivity and Publication Bias Analysis. We then used sensitivity analyses to evaluate the stability of the results of analysis considering all the included studies. No statistically significant change of the pooled estimated HRs for OS, CSS, and DFS/RFS was found when using the different effect models in meta-analysis of HRs from both UVA and MVA. In the leave-one-out sensitivity analyses, the result patterns were not obviously impacted by any single study for all analyses (data not shown). This indicated that our pooled results were 
TABLE 1: Main characteristics of eligible studies.

\begin{tabular}{|c|c|c|}
\hline Characteristics & $\begin{array}{c}\text { Number of } \\
\text { studies }\end{array}$ & $\begin{array}{c}\text { Number of } \\
\text { patients }\end{array}$ \\
\hline All eligible studies & 22 & 12829 \\
\hline \multicolumn{3}{|l|}{ Year of publication } \\
\hline 2014 & 3 & 1272 \\
\hline 2015 & 13 & 2951 \\
\hline 2016 & 6 & 8606 \\
\hline \multicolumn{3}{|l|}{ Endpoint used } \\
\hline OS & 18 & 11610 \\
\hline CSS & 6 & 1770 \\
\hline DFS/RFS & 13 & 8338 \\
\hline \multicolumn{3}{|l|}{ Method to get UVA HR } \\
\hline Reported & 16 & 10853 \\
\hline Estimated & 6 & 1976 \\
\hline \multicolumn{3}{|l|}{ Multivariate analysis } \\
\hline Performed & 21 & 12527 \\
\hline Unperformed & 1 & 302 \\
\hline \multicolumn{3}{|l|}{ Anatomic structure } \\
\hline Gastrointestinal tract & 17 & 11340 \\
\hline Nongastrointestinal tract & 5 & 1489 \\
\hline \multicolumn{3}{|l|}{ Tumor site } \\
\hline Esophagus & 3 & 892 \\
\hline Stomach & 3 & 1741 \\
\hline Colorectal & 11 & 8707 \\
\hline Liver & 2 & 660 \\
\hline Pancreas & 3 & 829 \\
\hline \multicolumn{3}{|l|}{ Stage } \\
\hline NMS & 11 & 9412 \\
\hline Mixed & 3 & 1789 \\
\hline MS & 8 & 1628 \\
\hline \multicolumn{3}{|l|}{ Ethnicity } \\
\hline Asian & 16 & 11285 \\
\hline Non-Asian & 6 & 1544 \\
\hline \multicolumn{3}{|l|}{ Cut-off value } \\
\hline$<3.0$ & 10 & 9272 \\
\hline$\geq 3.0$ & 12 & 3557 \\
\hline \multicolumn{3}{|l|}{ Treatment } \\
\hline With surgery & 18 & 12210 \\
\hline No surgery & 4 & 619 \\
\hline
\end{tabular}

OS: overall survival; CSS: cancer-specific survival; DFS: disease-free survival; RFS: recurrence-free survival; UVA: univariate analysis; HR: hazard ratio; NMS: nonmetastatic stage; MS: metastatic stage.

stable. Also, we assessed for publication bias of main results by the Egger test (Tables 2 and 3). No evidence of significant publication bias was observed except meta-analysis of MVA OS and MVA DFS. So, we further used the trim and fill method to validate the reliability of corresponding results. The pooled analysis incorporating the hypothetical studies continued to show statistically significant associations between poorer
MVA OS $(P<0.001)$ and MVA DFS $(P<0.001)$ with lower LMR. In summary, the results of sensitivity analyses and publication bias analyses supported the credibility of most of the evidence in this meta-analysis.

\section{Discussion}

Following the well-established and accepted concept that the pathogenesis of cancer is considered as an inflammationdriven malignancy [4], our current study for the first time comprehensively validated the clinical impact of pretreatment LMR in patients with digestive system tumor based on a large pool of clinical studies incorporating 12829 patients. We found a consistent harmful effect of decreased LMR on patient survival, including OS, CSS, and DFS/RFS in UVA pooled results. Such significant prognostic impact could be found among various disease subgroups, in organs with anatomic structure of gastrointestinal tract or nongastrointestinal tract, in Asian or non-Asian patients, regardless of metastatic stage and cut-off value, and in patients receiving surgery or not. With regard to the correlation between LMR and survival in MVA pooled results, similar negative effect of lower LMR was observed in total results of OS, CSS, and DFS/RFS. However, although subgroup analyses showed same trend of survival impact as total results, some results failed to reach significance. Moreover, substantial heterogeneity was observed in most results. This might be attributed to the fact that although HRs derived from MVA were results adjusted possible confounders at each study level, this still had an important limitation that was unavoidable: the different studies adjusted their MVA with different factors. This could impact the calculated multivariate HRs significantly and thus increase the risk of bias and heterogeneity. Therefore, caution must be exercised when interpreting the results. Meanwhile, whether LMR could act as an independent risk factor in digestive tumor still needs more studies to confirm.

However, the specific mechanism behind the association of higher LMR and favorable outcome of digestive system cancer patients still remains unclear. The unbalance between protumor and anticancer inflammatory status of hosts probably was the main reason $[40,41]$. In TME, the lymphocyte was usually regarded as one of the most crucial components of the host's cellular immunity [42] and the cellular basis of immunosurveillance and immunoediting against nascent tumor cells $[43,44]$ through induction of tumor cell apoptosis [45]. Hence, a low lymphocyte count might be responsible for a weak, insufficient immunologic reaction to tumor and thereby a worsened clinical outcome. On the contrary, the role of macrophages/monocytes in cancer development and progression remains controversial. Previous data showed a protective effect of tissue-specific macrophages cells [46, 47], such as Kupffer cells, which could eliminate circulating tumor cells. However, following the increasing focus on tumor-infiltrating macrophages (TIMs) derived from circulating monocytes, researchers found that, unlike common macrophages, TIMs enhanced tumor progression [48, 49]. The excessive production of TIMs can stimulate the growth of tumor cells, enhance neoangiogenesis, and thereby promote tumor cell migration and metastasis [50, 51]. TIMs can also 
TABLE 2: Meta-analysis of HRs from univariate survival analysis.

\begin{tabular}{|c|c|c|c|c|c|c|}
\hline Outcome & Groups & Number of studies & Model & HR (95\% CI) & $I^{2}(\%)$ & Egger test \\
\hline & All study & 18 & Fixed & $1.32[1.28-1.36]$ & 0 & 0.156 \\
\hline & Tumor site & & & & & \\
\hline & Esophagus & 2 & Fixed & 1.35 [1.22-1.49] & 0 & \\
\hline & Stomach & 3 & Fixed & 1.27 [1.19-1.35] & 0 & \\
\hline & Colorectal & 9 & Fixed & 1.33 [1.28-1.39] & 0 & \\
\hline & Liver & 2 & Fixed & $1.67[1.35-2.04]$ & 0 & \\
\hline & Pancreas & 2 & Fixed & $1.27[1.12-1.43]$ & 0 & \\
\hline & Anatomic structure & & & & & \\
\hline & $\mathrm{GT}$ & 14 & Fixed & 1.32 [1.28-1.37] & 0 & \\
\hline & Non-GT & 4 & Fixed & 1.35 [1.22-1.49] & 44 & \\
\hline & Stage & & & & & \\
\hline \multirow[t]{18}{*}{ OS } & NMS & 9 & Fixed & 1.33 [1.28-1.39] & 0 & \\
\hline & Mixed & 3 & Fixed & 1.28 [1.19-1.37] & 0 & \\
\hline & MS & 6 & Fixed & 1.35 [1.25-1.45] & 10 & \\
\hline & Ethnicity & & & & & \\
\hline & Asian & 13 & Fixed & 1.32 [1.28-1.37] & 0 & \\
\hline & Non-Asian & 5 & Fixed & 1.33 [1.22-1.45] & 0 & \\
\hline & Cut-offvalue & & & & & \\
\hline & $<3.0$ & 8 & Fixed & 1.32 [1.27-1.39] & 0 & \\
\hline & $\geq 3.0$ & 10 & Fixed & 1.32 [1.25-1.39] & 4 & \\
\hline & Treatment & & & & & \\
\hline & With surgery & 9 & Fixed & 1.32 [1.28-1.37] & 0 & \\
\hline & No surgery & 3 & Fixed & $1.33[1.22-1.45]$ & 0 & \\
\hline & All study & 6 & Random & $1.35[1.20-1.50]$ & 65 & 0.52 \\
\hline & Tumor site & & & & & \\
\hline & Colorectal & 3 & Fixed & 1.32 [1.18-1.47] & 29 & \\
\hline & Anatomic structure & & & & & \\
\hline & GT & 5 & Fixed & 1.39 [1.28-1.52] & 24 & \\
\hline & Stage & & & & & \\
\hline \multirow{16}{*}{ CSS } & Mixed & 2 & Random & 1.27 [1.04-1.54] & 70 & \\
\hline & MS & 3 & Fixed & 1.32 [1.18-1.47] & 29 & \\
\hline & Ethnicity & & & & & \\
\hline & Asian & 3 & Fixed & 1.49 [1.35-1.64] & 0 & \\
\hline & Non-Asian & 3 & Fixed & 1.21 [1.12-1.28] & 12 & \\
\hline & Cut-off value & & & & & \\
\hline & $<3.0$ & 3 & Random & 1.28 [1.11-1.49] & 80 & \\
\hline & $\geq 3.0$ & 3 & Fixed & 1.47 [1.27-1.69] & 0 & \\
\hline & All study & 13 & Random & $1.26[1.18-1.33]$ & 63 & 0.717 \\
\hline & Tumor site & & & & & \\
\hline & Stomach & 2 & Fixed & 1.22 [1.12-1.33] & 0 & \\
\hline & Colorectal & 7 & Fixed & 1.19 [1.11-1.28] & 46 & \\
\hline & Liver & 2 & Random & 1.39 [1.14-1.69] & 78 & \\
\hline & Anatomic structure & & & & & \\
\hline & GT & 10 & Fixed & 1.20 [1.18-1.25] & 29 & \\
\hline & Non-GT & 3 & Random & $1.41[1.23-1.61]$ & 58 & \\
\hline \multirow[t]{9}{*}{ DFS/RFS } & Stage & & & & & \\
\hline & NMS & 8 & Fixed & $1.22[1.18-1.27]$ & 46 & \\
\hline & MS & 4 & Random & 1.23 [1.03-1.49] & 81 & \\
\hline & Ethnicity & & & & & \\
\hline & Asian & 9 & Random & $1.27[1.18-1.35]$ & 71 & \\
\hline & Non-Asian & 4 & Fixed & 1.23 [1.12-1.37] & 32 & \\
\hline & Cut-off value & & & & & \\
\hline & $<3.0$ & 5 & Fixed & $1.27[1.20-1.32]$ & 11 & \\
\hline & $\geq 3.0$ & 8 & Random & $1.22[1.12-1.33]$ & 73 & \\
\hline
\end{tabular}

HR: hazard ratio; CI: confidence interval; OS: overall survival; CSS: cancer-specific survival; DFS: disease-free survival; RFS: recurrence-free survival; GT: gastrointestinal tract; NMS: nonmetastatic stage; MS: metastatic stage. 
TABLE 3: Meta-analysis of HRs from multivariate survival analysis.

\begin{tabular}{|c|c|c|c|c|c|c|}
\hline Outcome & Groups & Number of studies & Model & HR (95\% CI) & $I^{2}(\%)$ & Egger test \\
\hline & All study & 17 & Random & $1.21[1.31-1.38]$ & 73 & 0.028 \\
\hline & Tumor site & & & & & \\
\hline & Esophagus & 2 & Fixed & 1.25 [1.13-1.29] & 0 & \\
\hline & Stomach & 3 & Fixed & 1.11 [1.03-1.19] & 39 & \\
\hline & Colorectal & 8 & Fixed & 1.25 [1.19-1.30] & 36 & \\
\hline & Liver & 2 & Random & 1.37 [1.10-1.69] & 77 & \\
\hline & Pancreas & 2 & Random & $1.67[0.90-3.03]$ & 95 & \\
\hline & Anatomic structs & & & & & \\
\hline & GT & 13 & Fixed & $1.22[1.18-1.25]$ & 46 & \\
\hline & Non-GT & 4 & Random & $1.59[1.23-2.04]$ & 85 & \\
\hline & Stage & & & & & \\
\hline \multirow[t]{18}{*}{ OS } & NMS & 9 & Random & 1.33 [1.20-1.49] & 78 & \\
\hline & Mixed & 3 & Random & 1.12 [1.01-1.25] & 57 & \\
\hline & MS & 5 & Fixed & $1.32[1.23-1.41]$ & 27 & \\
\hline & Ethnicity & & & & & \\
\hline & Asian & 13 & Random & 1.27 [1.18-1.37] & 78 & \\
\hline & Non-Asian & 4 & Fixed & 1.35 [1.20-1.49] & 0 & \\
\hline & Cut-offvalue & & & & & \\
\hline & $<3.0$ & 7 & Random & 1.35 [1.19-1.33] & 83 & \\
\hline & $\geq 3.0$ & 10 & Fixed & 1.25 [1.16-1.35] & 63 & \\
\hline & Treatment & & & & & \\
\hline & With surgery & 13 & Random & 1.32 [1.28-1.37] & 79 & \\
\hline & No surgery & 4 & Fixed & 1.27 [1.18-1.35] & 0 & \\
\hline & All study & 5 & Random & $1.18[1.04-1.34]$ & 65 & 0.105 \\
\hline & Tumor site & & & & & \\
\hline & Colorectal & 2 & Fixed & 1.47 [1.19-1.82] & 0 & \\
\hline & Anatomic structs & & & & & \\
\hline & GT & 4 & Random & $1.23[1.02-1.52]$ & 72 & \\
\hline & Stage & & & & & \\
\hline \multirow{16}{*}{ CSS } & Mixed & 2 & Fixed & $1.06[0.99-1.22]$ & 26 & \\
\hline & MS & 2 & Fixed & 1.47 [1.19-1.82] & 0 & \\
\hline & Ethnicity & & & & & \\
\hline & Asian & 3 & Random & $1.20[0.96-1.52]$ & 77 & \\
\hline & Non-Asian & 2 & Random & 1.19 [0.95-1.49] & 12 & \\
\hline & Cut-off value & & & & & \\
\hline & $<3.0$ & 2 & Fixed & $1.12[1.04-1.22]$ & 44 & \\
\hline & $\geq 3.0$ & 3 & Random & $1.27[0.93-1.69]$ & 79 & \\
\hline & All study & 12 & Random & $1.26[1.16-1.38]$ & 83 & 0.025 \\
\hline & Tumor site & & & & & \\
\hline & Stomach & 2 & Fixed & $1.06[0.98-1.18]$ & 25 & \\
\hline & Colorectal & 6 & Random & 1.16 [1.09-1.25] & 52 & \\
\hline & Liver & 2 & Fixed & 1.37 [1.25-1.49] & 16 & \\
\hline & Anatomic structs & & & & & \\
\hline & GT & 9 & Fixed & 1.12 [1.09-1.16] & 46 & \\
\hline & Non-GT & 3 & Random & 1.59 [1.19-2.08] & 89 & \\
\hline \multirow[t]{9}{*}{ DFS/RFS } & Stage & & & & & \\
\hline & NMS & 8 & Random & 1.30 [1.15-1.47] & 85 & \\
\hline & MS & 3 & Random & $1.30[1.08-1.56]$ & 85 & \\
\hline & Ethnicity & & & & & \\
\hline & Asian & 8 & Random & 1.25 [1.11-1.41] & 88 & \\
\hline & Non-Asian & 4 & Random & 1.32 [1.10-1.59] & 32 & \\
\hline & Cut-off value & & & & & \\
\hline & $<3.0$ & 5 & Random & 1.45 [1.14-1.89] & 90 & \\
\hline & $\geq 3.0$ & 7 & Random & 1.18 [1.09-1.28] & 74 & \\
\hline
\end{tabular}

HR: hazard ratio; CI: confidence interval; OS: overall survival; CSS: cancer-specific survival; DFS: disease-free survival; RFS: recurrence-free survival; GT: gastrointestinal tract; NMS: nonmetastatic stage; MS: metastatic stage. 
TABLe 4: $P$ values of meta-regressions.

\begin{tabular}{lcc}
\hline Covariate & \multicolumn{2}{c}{ Meta-analysis of MVA HRs } \\
& OS & DFS/RFS \\
\hline $\begin{array}{l}\text { Anatomic structure } \\
\text { Tumor site }\end{array}$ & 0.015 & 0.043 \\
$\quad$ Esophagus & 0.118 & \\
Stomach & 0.016 & 0.003 \\
Colorectal & 0.094 & 0.015 \\
Liver & 0.752 & 0.018 \\
Pancreas & $\mathrm{DR}$ & 0.002 \\
Tumor stage & & $\mathrm{DR}$ \\
NMS & 0.983 & \\
Mix & 0.097 & 0.277 \\
MS & $\mathrm{DR}$ & $\mathrm{DR}$ \\
Ethnicity & 0.328 & 0.316 \\
Cut-off value & 0.120 & 0.637 \\
Beyond cut-off & 0.981 & 0.160 \\
Treatment & 0.772 & 0.720 \\
\hline
\end{tabular}

MVA: multivariate analysis; HR: hazard ratio; OS: overall survival; DFS: disease-free survival; RFS: recurrence-free survival; NMS: nonmetastatic stage; MS: metastatic stage; DR: dropped because of collinearity.

produce enzymes and inhibitors that regulate the digestion of the extracellular matrix and hence further favoring tumor invasion [52]. Moreover, these TIM-released soluble factors could also suppress the antitumor immune responses by making $T$ cell subsets lack cytotoxic function [53]. The circulating level of monocytes can reflect the formation or presence of TIMs, which explained why an elevated monocyte count confers a negative prognosis in patients with digestive system tumors.

The association of clinicopathological factors and LMR level was also reviewed in some studies retrieved in our analysis. Firstly, in terms of T category, Chan et al. [34] Huang and Feng [20], Hsu et al. [36], Lin et al. [22], and Li et al. [38] reported that lower LMR value was significantly correlated with an increased likelihood of higher degree of tumor infiltration (or larger tumor size) in esophageal cancer, gastric cancer, colorectal cancer, and liver cancer, respectively. Then, when considering $\mathrm{N}$ category, lower LMR was also significantly related to a higher risk of positive status of lymph nodes metastasis in esophageal cancer [20] and gastric cancer [36]. Moreover, in the study of Kozak et al. [21] about colorectal cancer, patients with lower LMR had significantly higher rates of worse stage, which was also consistent with the results of Hsu et al. [36] in gastric cancer and Li et al. [37] and Stotz et al. [31] in pancreatic cancer. These findings suggested that LMR could be a predictor of the clinicopathological features in some digestive system tumors.

This meta-analysis had several limitations that must be taken into account in the interpretation. Firstly, only summarized data rather than individual patient data could be used. Secondly, this analysis was constrained to studies published in English language only, and most of the literatures we brought into our review were from Asian countries (especially from
China) and were published in 2015. Although the "trim and fill" method has validated the reliability of the pooled results with publication bias, it still has to be noted that evaluation of publication bias could not be done in a robust manner with such few data points, and the statistical power of Egger's test to suspect publication bias was also kind of limited here. Thirdly, the cut-off values used by individual study varied from each other in the included studies, and the criteria method of selecting LMR cut-offs remained unclear. Last but not least, all included studies were retrospective singlecenter studies. Therefore it would be a little hard to control all kinds of factors that might affect patient survival and the level of inflammatory markers between two groups. Finally, obvious heterogeneity was observed for some of our analyses. Therefore, the random-effects model was used. Despite having tried several kinds of methods to figure out all sources of heterogeneity, the presence of heterogeneity might also result from many other factors, including age distribution, tumor size, and factors by which studies adjusted their multivariate analysis. In fact, since clinical and methodological diversity always occur in meta-analyses, statistical heterogeneity was inevitable [13]. Much more detailed data is needed to assess the heterogeneity in the future meta-regression. Of note, the results of meta-regressions in our study were only hypothesisgenerating rather than confirmatory, since the number of studies included in the analysis was kind of limited, and the possible influential factors found by exploratory metaregression may be subject to the false-positive conclusions because the false-positive rates could not be controlled completely [14].

\section{Conclusion}

In conclusion, this systematic review demonstrated the associations between lower LMR and poorer clinical outcomes in patients with digestive system tumors. LMR could be a convenient, easy-to-measure indicator for patients with a great clinical biological value in our future clinical practice.

\section{Competing Interests}

The authors declare that there is no conflict of interests regarding the publication of this paper.

\section{Authors' Contributions}

Jingwen Zhang and Lishan Chen contributed equally to this study.

\section{Acknowledgments}

The authors thank the members in W. Liao's Laboratory for advice and discussion. This work was supported by the National Natural Science Foundation of China (nos. 31271564 and 81472314 to Wangjun Liao), the Special Foundation for National Clinical Specialties of China (to The Department of Oncology, Nanfang Hospital), and the Team Program of 
Natural Science Foundation of Guangdong Province, China (no. S2011030003134).

\section{References}

[1] P. Friedl and S. Alexander, "Cancer invasion and the microenvironment: plasticity and reciprocity," Cell, vol. 147, no. 5, pp. 992-1009, 2011.

[2] T. L. Whiteside, "The tumor microenvironment and its role in promoting tumor growth," Oncogene, vol. 27, no. 45, pp. 59045912, 2008.

[3] R. R. Langley and I. J. Fidler, "The seed and soil hypothesis revisited-the role of tumor-stroma interactions in metastasis to different organs," International Journal of Cancer, vol. 128, no. 11, pp. 2527-2535, 2011.

[4] S. I. Grivennikov, F. R. Greten, and M. Karin, "Immunity, inflammation, and cancer," Cell, vol. 140, no. 6, pp. 883-899, 2010.

[5] A. Mantovani, P. Allavena, A. Sica, and F. Balkwill, "Cancerrelated inflammation,” Nature, vol. 454, no. 7203, pp. 436-444, 2008.

[6] E. F. Leitch, M. Chakrabarti, J. E. M. Crozier et al., "Comparison of the prognostic value of selected markers of the systemic inflammatory response in patients with colorectal cancer," British Journal of Cancer, vol. 97, no. 9, pp. 1266-1270, 2007.

[7] A. J. Templeton, M. G. McNamara, B. Šeruga et al., "Prognostic role of neutrophil-to-lymphocyte ratio in solid tumors: a systematic review and meta-analysis," Journal of the National Cancer Institute, vol. 106, no. 6, Article ID dju124, 2014.

[8] T. F. Nishijima, H. B. Muss, S. S. Shachar, K. Tamura, and Y. Takamatsu, "Prognostic value of lymphocyte-to-monocyte ratio in patients with solid tumors: a systematic review and metaanalysis," Cancer Treatment Reviews, vol. 41, no. 10, pp. 971-978, 2015.

[9] J.-J. Teng, J. Zhang, T.-Y. Zhang, S. Zhang, and B.-S. Li, "Prognostic value of peripheral blood lymphocyte-to-monocyte ratio in patients with solid tumors: a meta-analysis," OncoTargets and Therapy, vol. 9, pp. 37-47, 2015.

[10] D. Moher, A. Liberati, J. Tetzlaff, D. G. Altman, and P. Group, "Preferred reporting items for systematic reviews and metaanalyses: the PRISMA statement," PLoS Medicine, vol. 6, no. 7, Article ID e1000097, 2009.

[11] W. C. W. Wong, C. S. K. Cheung, and G. J. Hart, "Development of a quality assessment tool for systematic reviews of observational studies (QATSO) of HIV prevalence in men having sex with men and associated risk behaviours," Emerging Themes in Epidemiology, vol. 5, article 23, 2008.

[12] M. K. B. Parmar, V. Torri, and L. Stewart, "Extracting summary statistics to perform meta-analyses of the published literature for survival endpoints," Statistics in Medicine, vol. 17, no. 24, pp. 2815-2834, 1998.

[13] J. P. T. Higgins, S. G. Thompson, J. J. Deeks, and D. G. Altman, "Measuring inconsistency in meta-analyses," British Medical Journal, vol. 327, no. 7414, pp. 557-560, 2003.

[14] J. P. T. Higgins and S. G. Thompson, "Controlling the risk of spurious findings from meta-regression," Statistics in Medicine, vol. 23, no. 11, pp. 1663-1682, 2004.

[15] G. Knapp and J. Hartung, "Improved tests for a random effects meta-regression with a single covariate," Statistics in Medicine, vol. 22, no. 17, pp. 2693-2710, 2003.
[16] M. Egger, G. Davey Smith, M. Schneider, and C. Minder, "Bias in meta-analysis detected by a simple, graphical test," The British Medical Journal, vol. 315, no. 7109, pp. 629-634, 1997.

[17] J. P. T. Higgins and S. G. Thompson, "Quantifying heterogeneity in a meta-analysis," Statistics in Medicine, vol. 21, no. 11, pp. 15391558, 2002.

[18] Q. Deng, B. He, X. Liu et al., "Prognostic value of preoperative inflammatory response biomarkers in gastric cancer patients and the construction of a predictive model," Journal of Translational Medicine, vol. 13, no. 1, article 66, 2015.

[19] L.-H. Han, Y.-B. Jia, Q.-X. Song, J.-B. Wang, N.-N. Wang, and Y.F. Cheng, "Prognostic significance of preoperative lymphocytemonocyte ratio in patients with resectable esophageal squamous cell carcinoma," Asian Pacific Journal of Cancer Prevention, vol. 16, no. 6, pp. 2245-2250, 2015.

[20] Y. Huang and J.-F. Feng, "Low preoperative lymphocyte to monocyte ratio predicts poor cancer-specific survival in patients with esophageal squamous cell carcinoma," OncoTargets and Therapy, vol. 8, pp. 137-145, 2015.

[21] M. M. Kozak, R. von Eyben, J. S. Pai et al., "The prognostic significance of pretreatment hematologic parameters in patients undergoing resection for colorectal cancer," American Journal of Clinical Oncology, In press.

[22] Z.-X. Lin, D.-Y. Ruan, Y. Li et al., "Lymphocyte-to-monocyte ratio predicts survival of patients with hepatocellular carcinoma after curative resection," World Journal of Gastroenterology, vol. 21, no. 38, pp. 10898-10906, 2015.

[23] J.-S. Liu, Y. Huang, X. Yang, and J.-F. Feng, "A nomogram to predict prognostic values of various inflammatory biomarkers in patients with esophageal squamous cell carcinoma," American Journal of Cancer Research, vol. 5, no. 7, pp. 2180-2189, 2015.

[24] C. P. Neal, V. Cairns, M. J. Jones et al., "Prognostic performance of inflammation-based prognostic indices in patients with resectable colorectal liver metastases," Medical Oncology, vol. 32, no. 5, p. 144, 2015.

[25] K. Neofytou, E. C. Smyth, A. Giakoustidis et al., "The preoperative lymphocyte-to-monocyte ratio is prognostic of clinical outcomes for patients with liver-only colorectal metastases in the neoadjuvant setting," Annals of Surgical Oncology, vol. 22, no. 13, pp. 4353-4362, 2015.

[26] T. Ozawa, S. Ishihara, K. Kawai et al., "Impact of a lymphocyte to monocyte ratio in stage IV colorectal cancer," The Journal of Surgical Research, vol. 199, no. 2, pp. 386-392, 2015.

[27] Q. Qi, Y. Geng, M. Sun, P. Wang, and Z. Chen, "Clinical implications of systemic inflammatory response markers as independent prognostic factors for advanced pancreatic cancer," Pancreatology, vol. 15, no. 2, pp. 145-150, 2015.

[28] M. Shibutani, K. Maeda, H. Nagahara et al., "Prognostic significance of the lymphocyte-to-monocyte ratio in patients with metastatic colorectal cancer," World Journal of Gastroenterology, vol. 21, no. 34, pp. 9966-9973, 2015.

[29] A. Song, W. Eo, and S. Lee, "Comparison of selected inflammation-based prognostic markers in relapsed or refractory metastatic colorectal cancer patients," World Journal of Gastroenterology, vol. 21, no. 43, pp. 12410-12420, 2015.

[30] M. Stotz, M. Pichler, G. Absenger et al., "The preoperative lymphocyte to monocyte ratio predicts clinical outcome in patients with stage III colon cancer," British Journal of Cancer, vol. 110, no. 2, pp. 435-440, 2014.

[31] M. Stotz, J. Szkandera, T. Stojakovic et al., "The lymphocyte to monocyte ratio in peripheral blood represents a novel 
prognostic marker in patients with pancreatic cancer," Clinical Chemistry and Laboratory Medicine, vol. 53, no. 3, pp. 499-506, 2015.

[32] W.-W. Xiao, L.-N. Zhang, K.-Y. You et al., "A low lymphocyte-tomonocyte ratio predicts unfavorable prognosis in pathological T3N0 rectal cancer patients following total mesorectal excision," Journal of Cancer, vol. 6, no. 7, pp. 616-622, 2015.

[33] X. Zhou, Y. Du, J. Xu et al., “The preoperative lymphocyte to monocyte ratio predicts clinical outcomes in patients with stage II/III gastric cancer," Tumor Biology, vol. 35, no. 11, pp. 1165911666, 2014.

[34] J. C. Chan, D. L. Chan, C. I. Diakos et al., "The lymphocyteto-monocyte ratio is a superior predictor of overall survival in comparison to established biomarkers of resectable colorectal cancer," Annals of Surgery, 2016.

[35] A. Facciorusso, V. D. Prete, N. Crucinio, G. Serviddio, G. Vendemiale, and N. Muscatiello, "Lymphocyte-to-monocyte ratio predicts survival after radiofrequency ablation for colorectal liver metastases," World Journal of Gastroenterology, vol. 22, no. 16, pp. 4211-4218, 2016.

[36] J. T. Hsu, C. C. Wang, P. H. Le et al., "Lymphocyte-to-monocyte ratios predict gastric cancer surgical outcomes," The Journal of Surgical Research, vol. 202, no. 2, pp. 284-290, 2016.

[37] G. J. Li, H. W. Xu, J. J. Ji, F. Yang, and B. Q. Gao, "Prognostic value of preoperative lymphocyte-to-monocyte ratio in pancreatic adenocarcinoma," Journal of OncoTargets and Therapy, vol. 9, pp. 1085-1092, 2016.

[38] Y. Li, H. Jia, W. Yu et al., "Nomograms for predicting prognostic value of inflammatory biomarkers in colorectal cancer patients after radical resection," International Journal of Cancer, vol. 139, no. 1, pp. 220-231, 2016.

[39] S. J. Wu, Y. X. Lin, H. Ye, F. Y. Li, X. Z. Xiong, and N. S. Cheng, "Lymphocyte to monocyte ratio and prognostic nutritional index predict survival outcomes of hepatitis B virus-associated hepatocellular carcinoma patients after curative hepatectomy," Journal of Surgical Oncology, vol. 114, no. 2, pp. 202-210, 2016.

[40] K. Milne, C. Alexander, J. R. Webb et al., "Absolute lymphocyte count is associated with survival in ovarian cancer independent of tumor-infiltrating lymphocytes," Journal of Translational Medicine, vol. 10, no. 1, article 33, 2012.

[41] K. Haruki, H. Shiba, Y. Fujiwara et al., "Perioperative change in peripheral blood monocyte count may predict prognosis in patients with colorectal liver metastasis after hepatic resection," Journal of Surgical Oncology, vol. 106, no. 1, pp. 31-35, 2012.

[42] S. A. Rosenberg, "Progress in human tumour immunology and immunotherapy," Nature, vol. 411, no. 6835, pp. 380-384, 2001.

[43] G. P. Dunn, L. J. Old, and R. D. Schreiber, “The immunobiology of cancer immunosurveillance and immunoediting," Immunity, vol. 21, no. 2, pp. 137-148, 2004.

[44] H. Rabinowich, R. Cohen, I. Bruderman, Z. Steiner, and A. Klajman, "Functional analysis of mononuclear cells infiltrating into tumors: lysis of autologous human tumor cells by cultured infiltrating lymphocytes," Cancer Research, vol. 47, no. 1, pp. 173177, 1987.

[45] T. A. Zikos, A. D. Donnenberg, R. J. Landreneau, J. D. Luketich, and V. S. Donnenberg, "Lung T-cell subset composition at the time of surgical resection is a prognostic indicator in non-small cell lung cancer," Cancer Immunology, Immunotherapy, vol. 60, no. 6, pp. 819-827, 2011.

[46] I. J. Fidler and A. J. Schroit, "Recognition and destruction of neoplastic cells by activated macrophages: discrimination of altered self," Biochimica et Biophysica Acta (BBA)-Reviews on Cancer, vol. 948, no. 2, pp. 151-173, 1988.

[47] G. Heuff, H. S. A. Oldenburg, H. Boutkan et al., "Enhanced tumour growth in the rat liver after selective elimination of Kupffer cells," Cancer Immunology Immunotherapy, vol. 37, no. 2, pp. 125-130, 1993.

[48] E. Karnevi, R. Andersson, and A. H. Rosendahl, "Tumoureducated macrophages display a mixed polarisation and enhance pancreatic cancer cell invasion," Immunology and Cell Biology, vol. 92, no. 6, pp. 543-552, 2014.

[49] J. W. Pollard, "Tumour-educated macrophages promote tumour progression and metastasis," Nature Reviews Cancer, vol. 4, no. 1, pp. 71-78, 2004.

[50] A. Mantovani, B. Bottazzi, F. Colotta, S. Sozzani, and L. Ruco, "The origin and function of tumor-associated macrophages," Immunology Today, vol. 13, no. 7, pp. 265-270, 1992.

[51] J. W. Pollard, "Trophic macrophages in development and disease," Nature Reviews Immunology, vol. 9, no. 4, pp. 259-270, 2009.

[52] L. M. Coussens, C. L. Tinkle, D. Hanahan, and Z. Werb, "MMP9 supplied by bone marrow-derived cells contributes to skin carcinogenesis," Cell, vol. 103, no. 3, pp. 481-490, 2000.

[53] A. Sica, P. Allavena, and A. Mantovani, "Cancer related inflammation: the macrophage connection," Cancer Letters, vol. 267, no. 2, pp. 204-215, 2008. 


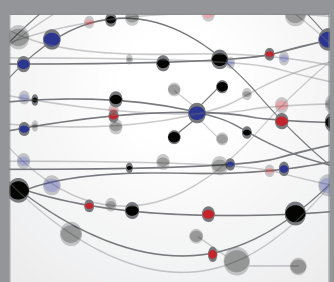

The Scientific World Journal
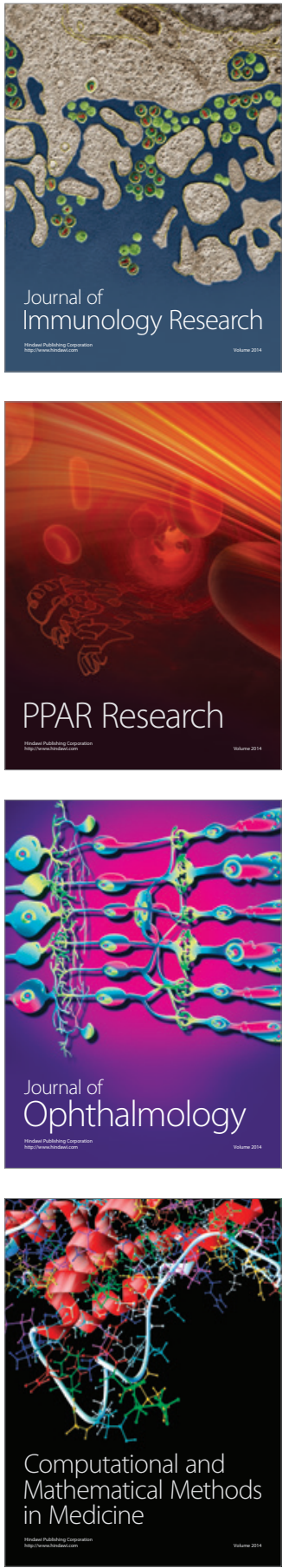

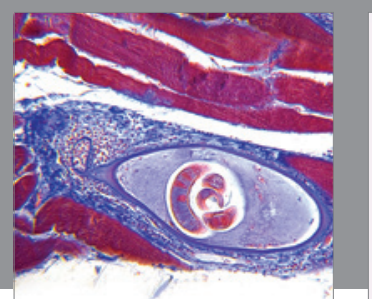

Gastroenterology Research and Practice

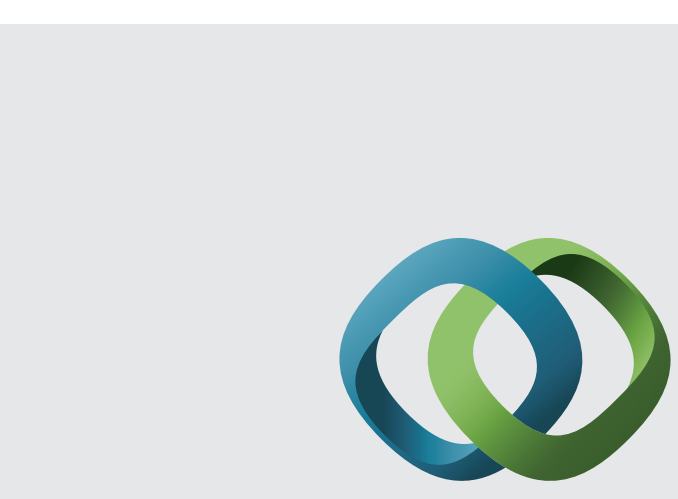

\section{Hindawi}

Submit your manuscripts at

http://www.hindawi.com
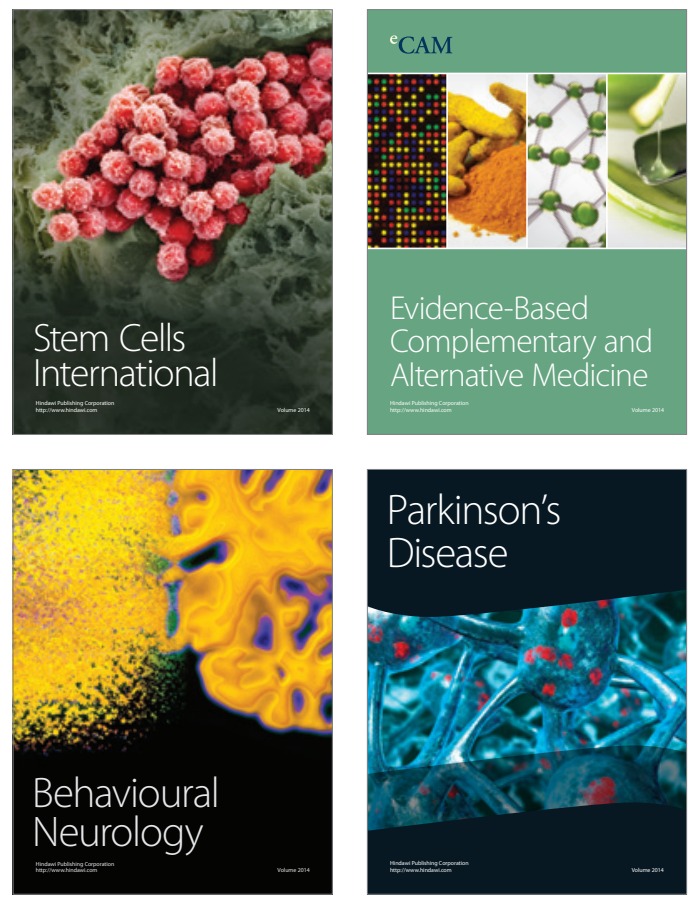
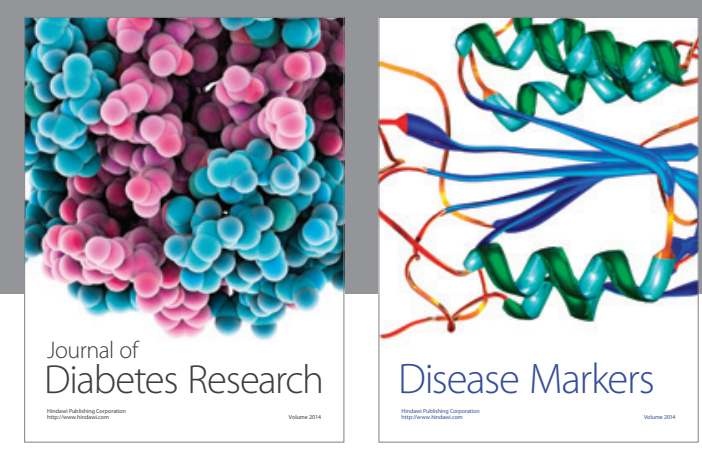

Disease Markers
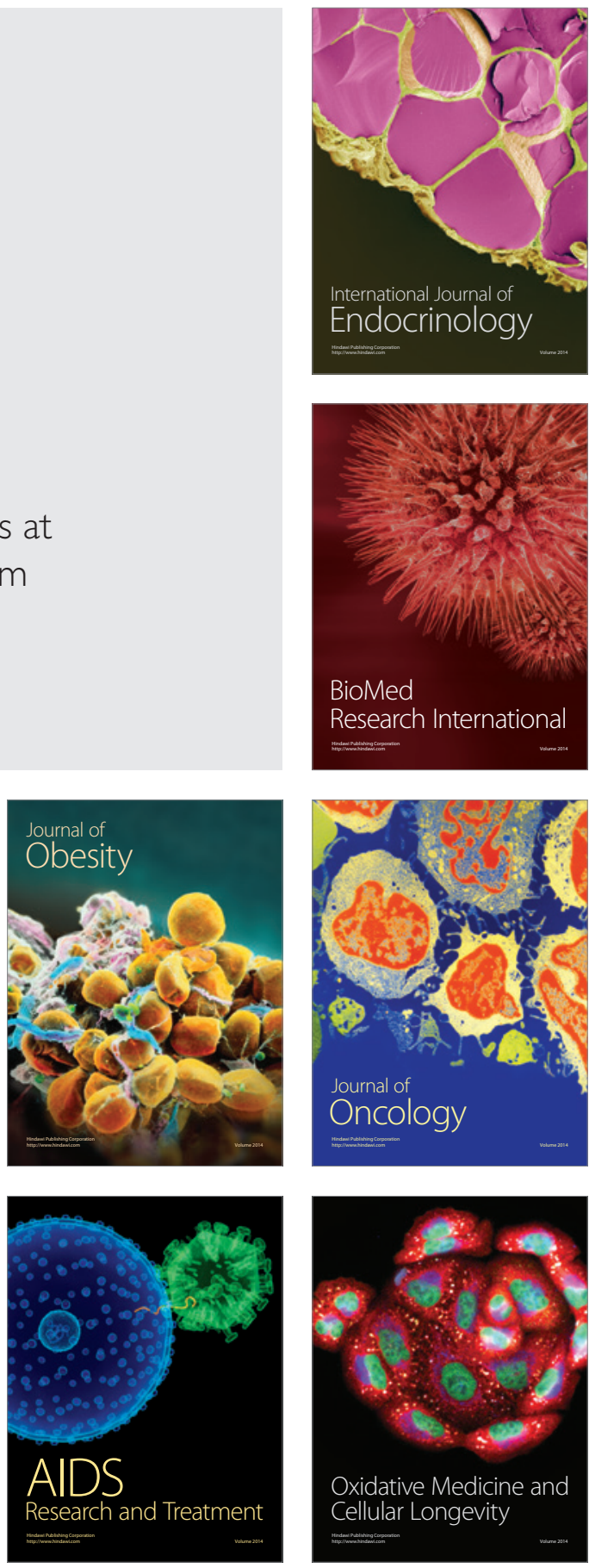